\title{
Free fatty acids and cardiovascular outcome: a Chinese cohort study on stable coronary artery disease
}

\author{
Hui-Wen Zhang ${ }^{\dagger}, X_{i} Z_{\text {hao }}^{\dagger}$, Yuan-Lin Guo, Cheng-Gang Zhu, Na-Qiong Wu, Jing Sun, Geng Liu, Qian Dong \\ and Jian-Jun Li*
}

\begin{abstract}
Background: Studies have revealed that plasma level of free fatty acids (FFAs) is related to cardiovascular disease. However, the role of FFAs in clinical outcomes of stable coronary artery disease (SCAD) has been less evaluated. This study, therefore, was to investigate the predictive value of FFAs in Chinese cohort with angiography-proven SCAD.
\end{abstract}

Methods: A total of 1670 consecutive patients with angina-like chest pain who underwent coronary angiography were enrolled and followed up for an average time of 36.9 months. The baseline characteristics were collected. Clinical and biochemical parameters of patients with or without cardiovascular events (CVEs) including all-cause death, non-fatal myocardial infarction, ischemic stroke and coronary revascularization were compared. According to the quartiles of baseline FFAs, patients were divided into four groups. The relationship of FFAs level and CVEs was evaluated.

Results: During the follow-up period, 146 patients suffered from CVEs. Patients with CVEs had elevated plasma level of FFAs than patients without CVEs $(P=0.002)$. Using the Cox proportional hazards model, the unadjusted hazard ratio (HR) for CVEs in the fourth FFAs quartile was 2.09 (95\% Cl:1.32-3.33; $P=0.002)$ and was $1.80(95 \% \mathrm{Cl}: 1.11-2.94 ; P=0$. 018) after adjustment for traditional cardiovascular risk factors when compared with that in the first FFAs quartile.

Conclusions: The present study on Chinese cohort with angiography-proven SCAD suggested that increased plasma level of FFAs was an independent risk factor of CVEs.

Keywords: Free fatty acids, Cardiovascular events, Stable coronary artery disease

\section{Background}

Coronary artery disease (CAD) is the leading cause of death in worldwide because of its adverse clinical outcomes. Free fatty acids (FFAs), also named as nonesterified fatty acids, have attracted considerable attention due to their predictive value in CAD [1,2]. FFAs are essential source of energy in human body originating from the adipose tissue, which provide $60 \%-$ $70 \%$ of adenosine triphosphate to the heart cells. Interestingly, previous data have indicated that elevated plasma FFAs level can enhance oxidative stress, subsequently resulting in vascular endothelial dysfunction [3-6] and

\footnotetext{
* Correspondence: lijianjun938@126.com

${ }^{\dagger}$ Equal contributors

Division of Dyslipidemia, State Key Laboratory of Cardiovascular Disease, Fu Wai Hospital, National Center for Cardiovascular Diseases, Chinese Academy of Medical Sciences and Peking Union Medical College, No 167 BeiLiShi Road, XiCheng District, Beijing 100037, China
}

inflammation [7, 8]. Meanwhile, FFAs have been shown to influence the metabolize of lipids and glucose, which may aggravate the development of atherosclerosis and insulin resistance $[9,10]$. Therefore, FFAs have been regarded as a risk marker of diabetes, obesity and cardiovascular diseases.

Although previous studies have suggested that plasma level of FFAs is related to the incidence and prognosis of cardiovascular disease [11-13], only two studies have revealed a positively predictive value of FFAs for clinical outcomes in patient with Stable CAD (SCAD) [12, 13]. In contrast, a study [14] on French cohort with male employees did not support the concept that FFAs is a marker for predicting the incidence of cardiovascular disease during a 15-year follow-up. The negative result was also found in the Quebec Cardiovascular Study cohort in participants free of cardiovascular disease [15]. 
Therefore, more researches are needed to determine whether FFAs are predictive for adverse cardiovascular events. In the present study, we enrolled a relatively large number of Chinese patients with SCAD to carefully examine the relationship between plasma level of FFAs and cardiovascular events (CVEs).

\section{Methods}

\section{Study population}

This protocol of the present study was complied with the Declaration of Helsinki, and was approved by the hospital ethics review board (Fu Wai Hospital and the National Center for Cardiovascular Diseases, Beijing, China). The informed consent was obtained from all the enrolled subjects.

From December 2011 through March 2013, a total of 2130 consecutive patients with angina-like chest pain referred for elective coronary angiography at our center were enrolled. SCAD was defined as described in our previous studies [16, 17], including patients with typical angina-like chest pain induced by exertion and/or positive treadmill exercise test, and had $50 \%$ diameter stenosis at least 1 of the 3 major coronary arteries or major branches assessed by elective coronary angiography. Exclusion criteria: acute coronary syndrome, heart failure (left ventricular ejection fraction, LVEF < 45\%), severe liver and/or renal insufficiency, infectious or systematic inflammatory disease, thyroid dysfunction, malignant disease and patients with unavailable FFAs variables. Finally, a total of 1670 patients were included. The baseline, clinical and laboratory parameters were collected from all enrolled patients. Hypertension was diagnosed with repeated blood pressure measurements $\geq 140 / 90 \mathrm{mmHg}$ or people who under anti-hypertensive drugs treatment. Type 2 diabetes was defined as fasting plasma glucose (FPG) $\geq 7.0 \mathrm{mmol} / \mathrm{L}$ and/or non-FPG $\geq 11.1 \mathrm{mmol} /$ Lin multiple examinations or under treatment with insulin or oral hypoglycemic agents. Dyslipidemia was defined as the presence of fasting plasma total cholesterol (TC) $\geq 5.2 \mathrm{mmol} / \mathrm{L}(200 \mathrm{mg} / \mathrm{dl})$ and/or triglyceride (TG) $\geq 1.7 \mathrm{mmol} / \mathrm{L}(150 \mathrm{mg} / \mathrm{dl})$. Body mass index was calculated as BMI $(\mathrm{kg} / \mathrm{m} 2)$ = body weight $(\mathrm{kg}) /$ body height $\left(\mathrm{m}^{2}\right)$.

\section{Laboratory analysis}

Blood samples of all the enrolled patients were obtained from cubital vein after a 12-h overnight fasting and collected into EDTA-containing tubes. All samples were subsequently stored at $-80{ }^{\circ} \mathrm{C}$ and analyzed immediately after thawing. The concentrations of plasma TC, TG, high-density lipoprotein cholesterol (HDL-C), highdensity lipoprotein cholesterol (LDL-C), and FFAs were measured using an automatic biochemistry analyzer
(Hitachi 7150, Tokyo, Japan). The concentrations of high-sensitive C-reactive protein (hs-CRP) were measured by immunoturbidometry (Beckmann Assay 360; Bera, CA, USA). Plasma hemoglobin A1c (HbA1c) levels were measured using the Tosoh G7 Automate HPLC Analyzer (TOSOH Bioscience, Japan).

\section{Definition of Events and Follow-up}

The predefined endpoint outcomes of the present study including all-cause mortality, non-fatal myocardial infarction (MI), ischemic stroke and coronary revascularization (CRV) including percutaneous coronary intervention (PCI) or coronary artery bypass graft surgery (CABG). Follow-up data of the enrolled patients were collected by the trained nurses who were blinded to the aim of this study. We performed standardized telephone interviews at 12,24, and 36 months. The follow-up time of each patient was calculated as the number of months from the enrollment till the last traceable hospital outpatient or inpatient record or telephone interview before March 2016, and was censored on the date of the endpoint events occurred.

\section{Statistical analysis}

Continuous variables were presented as mean \pm standard deviation (SD) or median with ranges and categorical variables as percentages (\%). Comparisons of continuous data between groups were performed with Student $t$ test or ANOVA as appropriate, and $\chi^{2}$ test Fisher's exact test were used for categorical data. The association of FFAs levels with events was described with calculated hazard ratios (HRs) and 95\% confidence intervals (CIs) using Cox proportional hazard models. Cumulative survival and event-free survival rates were estimated via the Kaplan-Meier method and compared using a log-rank test. Correlations between FFAs and other established cardiovascular risks variables were examined using Spearman or Pearson correlation coefficient when appropriate. A $P<0.05$ for two sided was considered as statistical significance. This statistical analysis was performed with SPSS 21.0 software (Chicago, IL).

\section{Results}

After an average follow-up time of 36.9 months, a total of 1506 patients finally completed the follow-up visit and 146 (9.7\%) patients suffered from CVEs, including all-cause death $(n=24)$, non-fatal MI $(n=20)$, ischemic stroke $(n=27)$, and CRV $(n=75)$. We compared the demographic characteristics between patients with CVEs and patients without CVEs as shown in Table 1. Data showed that patients with CVEs had a higher plasma level of FFAs than patients without CVEs $(P<0.05)$. Moreover, the group with CVEs presented a higher prevalence of hypertension, type 2 diabetes, and elevated level of FPG and HbA1c compared with that in patients 
Table 1 Demographic characteristics in patients with or without cardiovascular events (CVEs)

\begin{tabular}{|c|c|c|c|c|}
\hline Variables & $\begin{array}{l}\text { Total } \\
(n=1506)\end{array}$ & $\begin{array}{l}\text { Patients with CVEs } \\
(n=146)\end{array}$ & $\begin{array}{l}\text { Patients without CVEs } \\
(n=1360)\end{array}$ & $P$ value \\
\hline \multicolumn{5}{|l|}{ Baseline characteristics } \\
\hline Age (years) & $58.76 \pm 10.09$ & $58.65 \pm 10.04$ & $59.90 \pm 10.16$ & 0.23 \\
\hline Male (n,\%) & 1099(73\%) & $100(68.5 \%)$ & $999(73.5 \%)$ & 0.20 \\
\hline Family history of CAD $(n, \%)$ & 204(13.5) & $22(15.1)$ & $182(13.4)$ & 0.57 \\
\hline Hypertension (n,\%) & $971(64.5 \%)$ & 108(74\%) & $863(63.5 \%)$ & 0.01 \\
\hline Dyslipidemia $(n, \%)$ & $1165(77.4 \%)$ & 114(78.1\%) & $1051(77.3 \%)$ & 0.83 \\
\hline Diabetes (n,\%) & $431(28.6 \%)$ & $55(37.3 \%)$ & $376(27.6 \%)$ & 0.01 \\
\hline Current smoking $(n, \%)$ & $786(52.2 \%)$ & $66(45.2 \%)$ & $720(52.9 \%)$ & 0.08 \\
\hline BMI $\left(\mathrm{kg} / \mathrm{m}^{2}\right)$ & $25.57 \pm 3.16$ & $25.58 \pm 3.18$ & $25.51 \pm 2.96$ & 0.80 \\
\hline Lipid-lowering treatment $(n, \%)$ & $630(41.8 \%)$ & $54(37.0 \%)$ & $576(42.4 \%)$ & 0.21 \\
\hline Aspirin treatment $(n, \%)$ & 1480(98.3\%) & 143(97.9\%) & 1337(98.3\%) & 0.75 \\
\hline \multicolumn{5}{|l|}{ Laboratory parameters } \\
\hline TG $(\mathrm{mmol} / \mathrm{L})$ & $1.73 \pm 1.00$ & $1.78 \pm 1.17$ & $1.73 \pm 0.98$ & 0.54 \\
\hline $\mathrm{TC}(\mathrm{mmol} / \mathrm{L})$ & $4.14 \pm 1.13$ & $4.25 \pm 1.13$ & $4.13 \pm 1.13$ & 0.22 \\
\hline LDL-C (mmol/L) & $2.45 \pm 0.89$ & $2.53 \pm 0.95$ & $2.44 \pm 0.88$ & 0.27 \\
\hline $\mathrm{HDL}-\mathrm{C}(\mathrm{mmol} / \mathrm{L})$ & $1.08 \pm 0.26$ & $1.08 \pm 0.28$ & $1.08 \pm 0.26$ & 0.88 \\
\hline FFAs (mmol/L) & $0.43 \pm 0.19$ & $0.47 \pm 0.22$ & $0.42 \pm 0.19$ & 0.002 \\
\hline HbA1c (\%) & $6.41 \pm 1.14$ & $6.64 \pm 1.33$ & $6.39 \pm 1.12$ & 0.03 \\
\hline FPG (mmol/L) & $5.62 \pm 1.60$ & $5.92 \pm 1.85$ & $5.59 \pm 1.57$ & 0.04 \\
\hline LVEF(\%) & $62.53 \pm 8.61$ & $61.81 \pm 9.03$ & $62.72 \pm 8.54$ & 0.07 \\
\hline NT-pro-BNP (fmol/ml) & $728.27 \pm 532.25$ & $763.55 \pm 589.38$ & $713.71 \pm 494.94$ & 0.09 \\
\hline Creatine (umol/L) & $74.77 \pm 15.20$ & $76.35 \pm 15.98$ & $74.50 \pm 15.09$ & 0.12 \\
\hline hs-CRP (mg/L) & $2.88(0.01-17.69)$ & $3.04(0.01-13.73)$ & $2.87(0.01-17.69)$ & 0.12 \\
\hline cTnl (ng/ml) & $0.07(0.001-7.37)$ & $0.08(0.001-2.91)$ & $0.07(0.001-7.37)$ & 0.28 \\
\hline
\end{tabular}

Values are expressed as mean \pm SD, median with range, or $n$ (\%). SD: Standard deviation

$C A D$ coronary artery disease, $B M I$ body mass index, $T C$ total cholesterol, $T G$ triglyceride, $L D L-C$ low-density lipoprotein cholesterol, $H D L-C$ high-density lipoprotein cholesterol, HbA1c hemoglobin A1c, FPG fasting plasma glucose, LVEF left ventricular ejection fraction, NT-pro-BNP N-terminal-pro-brain natriuretic peptide, hs-CRP high sensitivity C-reactive protein, $c T n l$ cardiac troponin I

without CVEs $(P<0.05$, respectively). The mean age of the including subjects was 58.8 years old (range from 19 to 81 years old), and $73 \%$ of the enrolled participants were male patients. Given the wide age range from 19 to 81 years old of the participants, we compared the baseline characteristics according to age tertiles (the detailed characteristics were shown as Additional file 1: Table S1). Data showed no significant difference in the incidence of CVEs between age groups (tertile 1: $n=50,8.9 \%$ vs tertile 2: $n=48,9.6 \%$ vs tertile $3: n=48,10.7 \% ; P=0.64$ ). The mean value of FFAs was $0.43 \pm 0.19 \mathrm{mmol} / \mathrm{L}$ (ranged from 0.03 to $1.40 \mathrm{mmol} / \mathrm{L}$ ) in the enrolled population. According to the quartiles of plasma level of FFAs, the 1506 patients were divided into four groups, quartile $1(\leq 0.3 \mathrm{mmol} / \mathrm{L}$, $n=403)$, quartile $2(0.31-0.40 \mathrm{mmol} / \mathrm{L}, n=364)$, quartile $3(0.41-0.53 \mathrm{mmol} / \mathrm{L}, n=391)$, quartile $4(>0.53 \mathrm{mmol} / \mathrm{L}$, $n=348)$. The baseline characteristics and laboratory parameters of the included subjects according to FFAs quartiles were shown in Table 2. The group in the fourth quartile of FFAs appeared to have higher levels of TG, TC, BMI, FPG, HbA1c, hs-CRP and lower level of HDL-C $(P<0.05$, respectively) when compared with the first quartile of FFAs. Patients in the fourth quartile of FFAs also showed higher prevalence of type 2 diabetes, dyslipidemia and hypertension $(P<0.05$, respectively) when compared with the first quartile of FFAs. There was no significant difference in proportion of lipid-lowering drugs treatment $(P>0.05)$ among the four groups. There was a significantly increased incidence of CVEs with the elevation of plasma FFAs level (quartile $1: n=28,6.9 \%$ vs quartile 2 : $n=31,8.5 \%$ vs quartile $3: n=38,9.7 \%$ vs quartile 4 : $n=49,14.1 \% ; P=0.008)$.

Data from our study showed a strong association between baseline plasma level of FFAs and incidence of all endpoint events as established in Table 3. The unadjusted HR for CVEs in the fourth FFAs quartile was 2.09 (95\% CI 1.32-3.33; $P=0.002)$ when compared with subjects in the first FFA quartile. The multivariate 
Table 2 Demographic characteristics stratified by FFAs quartiles (mmol/L)

\begin{tabular}{|c|c|c|c|c|c|}
\hline Variables & $\begin{array}{l}\text { Quartile } 1 \\
(\leq 0.30) \\
(n=403)\end{array}$ & $\begin{array}{l}\text { Quartile 2 } \\
(0.31-0.40) \\
(n=364) \\
\end{array}$ & $\begin{array}{l}\text { Quartile 3 } \\
(0.41-0.53) \\
(n=391)\end{array}$ & $\begin{array}{l}\text { Quartile } 4 \\
(>0.53) \\
(n=348)\end{array}$ & $P$ Value \\
\hline \multicolumn{6}{|l|}{ Baseline characteristics } \\
\hline Age (years) & $58.65 \pm 9.70$ & $58.14 \pm 10.15$ & $58.50 \pm 10.31$ & $59.90 \pm 10.16$ & 0.15 \\
\hline Male (n,\%) & $292(72.5 \%)$ & $283(77.7 \%)$ & $295(75.4 \%)$ & $229(65.8 \%)$ & 0.002 \\
\hline Family history of CAD $(n, \%)$ & $49(12.2 \%)$ & $45(12.4 \%)$ & $62(16.0 \%)$ & 48(13.8\%) & 0.41 \\
\hline Hypertension (n,\%) & $226(56.5 \%)$ & $228(63.7 \%)$ & $269(69.0 \%)$ & $248(71.3 \%)$ & $<0.001$ \\
\hline Dyslipidemia $(n, \%)$ & $293(73.3 \%)$ & $267(74.8 \%)$ & $315(80.6 \%)$ & 290(83.3\%) & 0.001 \\
\hline Diabetes (n,\%) & $72(18 \%)$ & $77(21.6 \%)$ & $127(32.5 \%)$ & 115(33\%) & $<0.001$ \\
\hline Current smoking $(n, \%)$ & $220(55 \%)$ & 193(53.6\%) & $208(53.3 \%)$ & $165(47.6 \%)$ & 0.22 \\
\hline BMI $\left(\mathrm{kg} / \mathrm{m}^{2}\right)$ & $25.00 \pm 3.24$ & $25.54 \pm 2.96$ & $25.76 \pm 3.19$ & $26.02 \pm 3.13$ & $<0.001$ \\
\hline Lipid-lowering treatment $(n, \%)$ & $170(42.2 \%)$ & $154(42.3 \%)$ & $167(42.7 \%)$ & 139(39.9\%) & 0.88 \\
\hline Aspirin treatment $(n, \%)$ & 402(99.8\%) & $354(97.3 \%)$ & $385(98.5 \%)$ & $339(97.4 \%)$ & 0.03 \\
\hline \multicolumn{6}{|l|}{ Laboratory parameters } \\
\hline TG $(\mathrm{mmol} / \mathrm{L})$ & $1.48 \pm 0.60$ & $1.75 \pm 1.00$ & $1.84 \pm 1.02$ & $1.91 \pm 1.23$ & $<0.001$ \\
\hline $\mathrm{TC}(\mathrm{mmol} / \mathrm{L})$ & $4.03 \pm 1.00$ & $4.03 \pm 0.94$ & $4.17 \pm 1.06$ & $4.36 \pm 1.47$ & 0.002 \\
\hline LDL-C (mmol/L) & $2.43 \pm 0.88$ & $2.36 \pm 0.80$ & $2.47 \pm 0.90$ & $2.55 \pm 0.96$ & 0.07 \\
\hline $\mathrm{HDL}-\mathrm{C}(\mathrm{mmol} / \mathrm{L})$ & $1.08 \pm 0.26$ & $1.05 \pm 0.23$ & $1.07 \pm 0.26$ & $1.12 \pm 0.28$ & 0.02 \\
\hline FFAs (mmol/L) & $0.23 \pm 0.06$ & $0.35 \pm 0.03$ & $0.46 \pm 0.04$ & $0.69 \pm 0.17$ & $<0.001$ \\
\hline $\mathrm{HbA} 1 \mathrm{c}(\%)$ & $6.17 \pm 0.89$ & $6.19 \pm 1.03$ & $6.55 \pm 1.20$ & $6.77 \pm 1.32$ & $<0.001$ \\
\hline FPG (mmol/L) & $5.22 \pm 1.13$ & $5.33 \pm 1.31$ & $5.75 \pm 1.62$ & $6.21 \pm 2.06$ & $<0.001$ \\
\hline LVEF(\%) & $62.53 \pm 8.94$ & $62.59 \pm 8.83$ & $62.79 \pm 8.12$ & $62.18 \pm 8.57$ & 0.82 \\
\hline NT-pro-BNP (fmol/ml) & $712.62 \pm 463.92$ & $691.97 \pm 409.19$ & $709.71 \pm 516.94$ & $805.07 \pm 703.82$ & 0.07 \\
\hline Creatine (umol/L) & $74.17 \pm 14.65$ & $75.42 \pm 14.07$ & $75.04 \pm 14.73$ & $74.49 \pm 17.35$ & 0.30 \\
\hline hs-CRP (mg/L) & $2.31(0.01-15.15)$ & $2.61(0.01-16.51)$ & $3.42(0.01-17.17)$ & $3.22(0.01-17.69)$ & $<0.001$ \\
\hline cTnl (ng/ml) & $0.08(0.001-6.03)$ & $0.06(0.001-6.01)$ & $0.09(0.001-7.37)$ & $0.07(0.001-4.46)$ & 0.57 \\
\hline Cardiovascular events $(n, \%)$ & 28(6.9\%) & $31(8.5 \%)$ & $38(9.7 \%)$ & $49(14.1 \%)$ & 0.008 \\
\hline
\end{tabular}

Values are expressed as mean \pm SD, median with range, or $n$ (\%). SD: Standard deviation

$C A D$ coronary artery disease, $B M I$ body mass index, $T C$ total cholesterol, $T G$ triglyceride, $L D L-C$ low-density lipoprotein cholesterol, $H D L-C$ high-density lipoprotein cholesterol, $H b A 1 c$ hemoglobin A1c, FPG fasting plasma glucose, $L V E F$ left ventricular ejection fraction, NT-pro-BNP N-terminal-pro-brain natriuretic peptide, hs-CRP high sensitivity C-reactive protein, $C T n l$ cardiac troponin I

analysis showed that plasma level of FFAs was an independent predictor for CVEs after adjusting for age, gender (HR:2.06, 95\% CI:1.29-3.27; $P=0.002$ ). FFAs level remained to be an independent predictor of adverse outcomes after adjusted for potential confounders including hypertension, diabetes, BMI, current smoking, and family history of CAD (HR:1.82, 95\% CI:1.13-2.93; $P=0.014)$. Further adjusted for HbA1c, TC, TG, HDL$\mathrm{C}$, hs-CRP and cardiac troponin I. the result remained significant (HR: 1.80, 95\% CI: 1.11-2.94; $P=0.018$ ). In the study group, the incidence of CVEs in the fourth FFAs quartile was 4.96 per 100 person years compared to 2.46 per 100 person years in the first quartile $(P<0.001)$. As shown in Table 4, we additionally evaluated the relationship between plasma FFAs level and all-cause death, non-fatal MI, stroke and CRV respectively. Data indicated that FFAs level was also an independent risk factor of all-cause death (adjusted HR:4.11, 95\% CI:1.23-13.73; $P=0.02$ ), while there was no significant association between FFAs and nonfatal MI (adjusted HR:1.71, 95\% CI:0.40-7.39; $P=0.47$ ), stroke (adjusted HR:1.24, 95\% CI:0.43-7.55; $P=0.68)$ in the present study. The unadjusted HR of fourth quartile of FFAs (first quartile as reference) in predicting for CRV was 1.92 (95\% CI:1.02-3.93; $P=0.03)$, however, this association disappeared when further adjusted for conventional risk factors (adjusted HR:1.65, 95\% CI:0.82-3.33; $P=0.16$ ).

The Kaplan-Meier curves for cumulative event-free survival based on the quartiles of baseline FFAs levels were presented in Fig. 1, the highest quartile of plasma FFAs was positively associated with increase of CVEs when compared with the lowest quartile. We further investigated the relation between FFAs with established 
Table 3 Risk of cardiovascular events based on the quartiles of FFAs (first quartile as reference)

\begin{tabular}{|c|c|c|c|c|}
\hline & $\begin{array}{l}\text { Quartile } 1 \\
(\leq 0.30, n=403)\end{array}$ & $\begin{array}{l}\text { Quartile } 2 \\
(0.31-0.40, n=364)\end{array}$ & $\begin{array}{l}\text { Quartile } 3 \\
(0.41-0.53, n=391)\end{array}$ & $\begin{array}{l}\text { Quartile } 4 \\
(>0.53, n=348)\end{array}$ \\
\hline Incident cases & 28 & 31 & 38 & 49 \\
\hline$P$ value & - & 0.63 & 0.16 & 0.001 \\
\hline Person-years & 1138.92 & 1024.78 & 1138.51 & 987.50 \\
\hline Incidence/100 person-years & 2.46 & 3.03 & 3.34 & 4.96 \\
\hline$P$ value & - & 0.25 & 0.03 & $<0.001$ \\
\hline Univariate & 1.00 (Ref.) & $1.24(0.74-2.06)$ & $1.42(0.87-2.32)$ & $2.09(1.32-3.33)$ \\
\hline$P$ value & - & 0.41 & 0.16 & 0.002 \\
\hline Model $1^{\mathrm{a}}$ & 1.00 (Ref.) & $1.26(0.75-2.09)$ & $1.15(0.88-2.33)$ & $2.06(1.29-3.27)$ \\
\hline$P$ value & - & 0.38 & 0.15 & 0.002 \\
\hline Model $2^{b}$ & 1.00 (Ref.) & $1.20(0.72-2.01)$ & $1.29(0.79-2.13)$ & $1.82(1.13-2.93)$ \\
\hline$P$ value & - & 0.49 & 0.31 & 0.014 \\
\hline Model $3^{c}$ & 1.00 (Ref.) & $1.16(0.69-1.96)$ & $1.30(0.79-2.15)$ & $1.80(1.11-2.94)$ \\
\hline$P$ value & - & 0.58 & 0.31 & 0.018 \\
\hline
\end{tabular}

HRs Hazard ratios, $\mathrm{Cl}$ Confidence interval

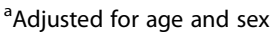

${ }^{\mathrm{b}}$ Additionally adjusted for hypertension, diabetes, body mass index, current smoking, and family history of coronary artery disease

${ }^{\mathrm{C}}$ Additionally adjusted for hemoglobinA1c, total cholesterol, triglycerides, high-density lipoprotein cholesterol, high sensitivity C-reactive protein, and cardiac

Troponin I

and emerging risk factors of cardiovascular disease. For the Spearman correlations analysis, furthermore, plasma level of FFAs was positively correlated with FPG $(r=0.239, P<0.001)$, HbA1c $(r=0.215, P<0.001)$, TC $(r=0.149, P<0.001)$ and hs-CRP $(r=0.127, P<0.001)$.

\section{Discussion}

Data from our relatively large prospective Chinese cohort mainly revealed that plasma level of FFAs was an independent risk maker for evaluating future CVEs predefined as all-cause mortality, MI, ischemic stroke and CRV. Our data suggested that higher FFAs level predicted for a worse outcome in patients with angiography-proven SCAD and the observed positive association of FFAs with CVEs did not alter even after adjustment of conventional cardiovascular risk factors.

Table 4 Univariate and multivariate Cox hazard for different endpoint based on the fourth quartile of FFAs (first quartile as reference)

\begin{tabular}{lllllll}
\hline & Univariate & & & Multivariate & \\
\cline { 2 - 3 } \cline { 6 - 7 } \cline { 6 - 7 } & HRs $(95 \% \mathrm{Cls})$ & $P$ value & & HRs $(95 \% \mathrm{Cls})$ & $P$ value \\
\hline All-cause death & $3.55(1.14-10.99)$ & 0.03 & & $4.11(1.23-13.73)$ & 0.02 \\
No-fatal MI & $2.22(0.59-9.50)$ & 0.22 & & $1.71(0.40-7.39)$ & 0.47 \\
Stroke & $1.71(0.40-7.39)$ & 0.40 & & $1.24(0.43-7.55)$ & 0.68 \\
CRV & $1.92(1.02-3.93)$ & 0.03 & & $1.65(0.82-3.33)$ & 0.16 \\
\hline
\end{tabular}

HRs Hazard ratios, Cl Confidence interval, MI Myocardial infarction, CRV Coronary revascularization

Multivariate model adjusted for age, sex, hypertension, diabetes, body mass index, current smoking, and family history of coronary artery disease, hemoglobinA1c, total cholesterol, triglycerides, high-density lipoprotein cholesterol, high sensitivity C-reactive protein and cardiac Troponin I
Although FFAs have been uncovered for many years, the investigations of their value in clinical diagnosis and prognosis of metabolic disorders are still of great interests. FFAs were mainly released from the storage in adipose tissue, which were originally considered to be of great importance to supply physiological source of energy for human body [2]. The physiological functions of FFAs included cell membrane formation, cellular signal transduction and regulate glucose metabolism $[3,4]$.

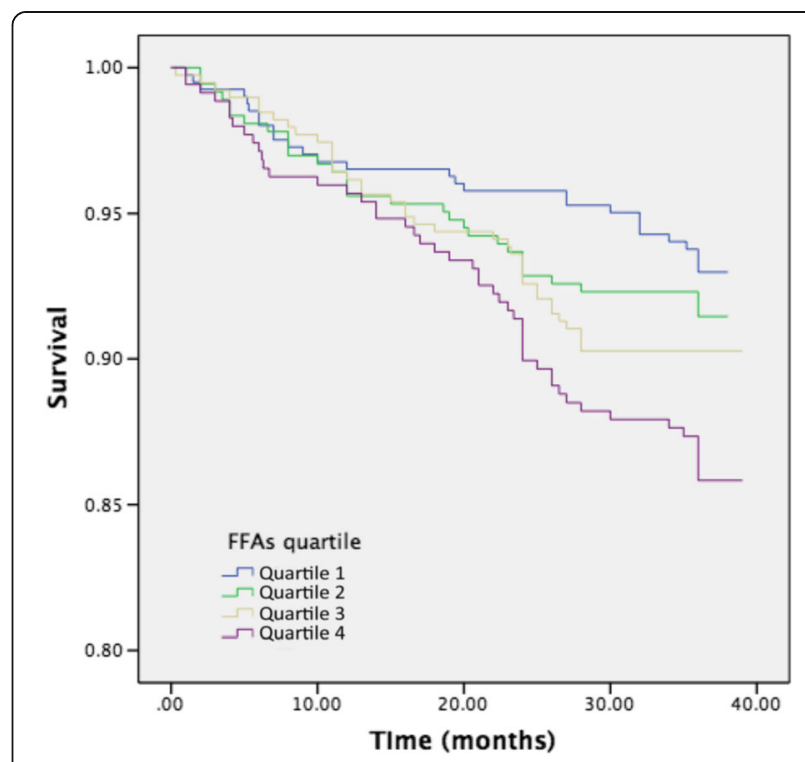

Fig. 1 The Kaplan-Meier curves for cumulative event-free survival based on the quartiles of baseline FFAs levels 
Therefore, several potential mechanisms may account for the findings in the present study. On the one hand, FFAs induced oxidative stress and inflammatory reaction $[5,6]$, leading to an acceleration in the rupture of atherosclerotic lesions and the progress atherosclerosis in patients with CAD. It was also reported that FFAs could induce inflammation and injury vascular endothelial cells [7, 8], which was essential etiology in the incidence and development of artery atherosclerosis [18-21]. In addition, elevated plasma level of FFAs could influence glucose production [9], which was demonstrated to be a risk marker for insulin resistant. In the previous studies, FFAs were revealed to be associated with metabolic syndrome as obesity and type 2 diabetes [22-24]. In our study, we also found positive association between FFAs and FPG $(r=0.239, P<0.05)$, HbA1c $(r=0.215, P<0.05)$, which was in consistent with previous studies [22-24]. Moreover, high level of FFAs was also found to play a role in arrhythmia [25, 26] and myocardial dysfunction [27-29]. Thereby, studies on the role of FFAs in predicting for atherosclerotic cardiovascular disease are benefit for improving strategies for prevention and therapy in CAD.

Several studies have investigated the relationship between FFAs and CAD previously, the conclusions were still in controversial. More recently, a prospective study of 3300 white individuals reported by Pliz et al. [12] have revealed that FFAs are independently associated with cardiac mortality in patients with angiographic CAD (HR:1.94, 95\% CI:1.32-2.85; $P=0.001$ ), stable CAD (HR:1.88, 95\% CI:1.19-2.96; $P=0.007$ ) and unstable CAD (HR:2.22, 95\% CI:1.03-4.77; $P=0.041$ ). Furthermore, Pliz [30] have also suggested that elevated level of FFAs is an independent risk factor for future sudden cardiac death (HR:1.76, 95\% CI:1.03-3.00; $P=0.038$ ) in 2231 angiographic CAD patients after 6.85-year followup. In addition, data from Breitling [13] included 1206 participants with 8 years of follow-up revealed an increased mortality associated with high levels of FFAs in SCAD (HR:2.071, 95\% CI:1.066-4.021; $P=0.032$ ). Michael et al. [31] showed that elevated plasma concentrations of FFAs were associated with cardiovascular and non-cardiovascular mortality in 4707 old subjects ( $\geq 65$ years) from The Cardiovascular Health Study. Conversely, another prospective study [14] showed no significant association between FFAs and CVD mortality after adjustment for traditional risk factors in 4589 middle-aged men (mean age 48.8 years old). This result could be attributed to that plasma concentration of FFAs was influenced by factors such as stress, nutritional and smoking status of the enrolled patients since all the participants were men. Another hypothesis was that the metabolism of FFAs which could influence the contribution the cardiovascular risk might vary among different individuals [14]. A negative result was also found in a prospective research of Quebec Cardiovascular Study cohort [15] which suggested that elevated plasma FFAs concentrations were not associated with an increased risk of ischemia heart disease, it could be due to the fact that participants had normal range of plasma level of FFAs and the relatively small sample size of this study. Apparently, more researches are needed for performing FFAs-related outcomes. However, data regarding the role of FFAs in predicting cardiovascular outcome in Chinese patients with SCAD are not available. Here, in the present study, we conducted the relatively large prospective cohort in Chinese patients with SCAD, data showed that plasma level of FFAs was higher in patients with CVEs compared with patients without CVEs, and a significantly elevated incidence of CVEs was observed as the level of FFAs increased. The present study validated that FFAs were independently associated with CVEs even adjusted for established risk factors such as gender, current smoking, hypertension, diabetes and lipids level (adjusted HR 1.80, 95\% CI: $1.11-2.94 ; P=0.018$ ) in patients with SCAD among Chinese cohort. We also revealed a significant value of FFAs in predicting for allcause death (adjusted HR:4.11, 95\% CI:1.23-13.73; $P=0.022$ ). Our study, obviously, provided novel information with regard to the role of FFAs in SCAD. In addition, measurement of plasma FFAs levels is convenient and at less costs. Therefore, data from our study provided important clue to make more effective strategies for secondary prevention and therapy in patients with SCAD.

There were several limitations in the present study. Patients from a single-center study was the first limitation which may introduce a potential selection bias. Secondly, we have only measured the plasma level of FFAs for a single examination, since plasma concentration of FFAs could be influenced by the nutritional state, stress, physical activity and period of disease. And the change of FFAs level over the development of SCAD was not clear. Therefore, once measurement of fasting FFA may not accurately reflect the variations of FFAs in circulation. Then, the follow-up time of this study was relatively short. Therefore, further investigations with larger population and longer follow-up time are needed to confirm our study.

\section{Conclusions}

In summary, data from our study validated that plasma level of FFAs was an independent risk factor for predicting CVEs in Chinese patients with SCAD. Elevated FFAs levels were related to a worse prognosis in these subjects. Our study suggested the potential of FFA-target strategies may improve the prognosis in patients with SCAD. Further studies are needed to confirm our study. 


\section{Additional file}

Additional file 1: Table S1. Demographic characteristics stratified by age tertiles (years). (DOCX $104 \mathrm{~kb}$ )

\section{Abbreviations}

95\% Cls: 95\% confidence intervals; BMI: Body mass index; CABG: Coronary artery bypass graft surgery; CRV: Coronary revascularization; CTnl: cardiac troponin I; CVEs: Cardiovascular events; FFAs: Free fatty acids; FPG: Fasting plasma glucose; HbA1c: Hemoglobin A1c; HDL-C: High-density lipoprotein cholesterol; HRs: Hazard ratios; hs-CRP: High sensitivity C-reactive protein; LDL-C: Low-density lipoprotein cholesterol; MI: Myocardial infarction; PCl: Percutaneous coronary intervention; SCAD: Stable coronary artery disease; TC: Total cholesterol; TG: Triglyceride

\section{Acknowledgments}

None.

\section{Funding}

This study was partly supported by National Natural Scientific Foundation $(81,241,121,81,241,121)$, Capital Special Foundation of Clinical Application Research (Z121107001012015), Capital Health Development Fund $(2,011,400,302,201,614,035)$, Beijing Natural Scientific Foundation (7131014), and CAMS Major Collaborative Innovation Project (2016-I2M-1-011), Youth Foundation of Peking Union Medical College (3332016023).

\section{Availability of data and materials}

The datasets analyzed during the present study are available from the corresponding author on reasonable request.

\section{Authors' contributions}

ZHW, ZX analyzed the data and wrote the manuscript. LJJ conceived the study and contributed to reviewing/editing the manuscript. GYL, ZCG, WNQ, SJ, LG, and DQ contributed to collecting data and reviewing the manuscript. All authors have read and approved the final manuscript.

\section{Ethics approval and consent to participate}

This protocol of the present study was complied with the Declaration of Helsinki, and was approved by the hospital ethics review board (Fu Wai Hospital and the National Center for Cardiovascular Diseases, Beijing, China). The informed consent was obtained from all the enrolled subjects.

\section{Consent for publication}

Not applicable.

\section{Competing interests}

The authors declare that they have no competing interests.

Received: 8 April 2017 Accepted: 13 June 2017

Published online: 26 June 2017

\section{References}

1. Pilz S, Marz W. Free fatty acids as a cardiovascular risk factor. Clin Chem Lab Med. 2008:46(4):429-34.

2. Baum SJ, Kris PM. Fatty acids in cardiovascular health and disease: a comprehensive update. J Clin Lipidol. 2012;6(3):216-34.

3. Steinberg HO, Tarshoby M, Monestel R, Hook G, Cronin J, Johnson A, et al. Flevated circulating free fatty acid levels impair endothelium-dependent vasodilation. J Clin Invest. 2011;100(5):1230-9.

4. Mathew M, Tay E, Cusi K. Elevated plasma free fatty acids increase cardiovascular risk by inducing plasma biomarkers of endothelial activation, myeloperoxidase and PAI-1 in healthy subjects. Cardiovasc Diabetol. 2010; 9(1):1-9.

5. Steinberg HO, Paradisi G, Hook G, Crowder K, Cronin J, Baron AD. Free fatty acid elevation impairs insulin-mediated vasodilation and nitric oxide production. Diabetes. 2000;49(7):1231-8

6. Hufnagel B, Dworak M, Soufi M, Mester Z, Zhu Y, Schaefer JR, et al. Unsaturated fatty acids isolated from human lipoproteins activate protein phosphatase type 2Cbeta and induce apoptosis in endothelial cells. Atherosclerosis. 2005;180(2):245-54.
7. Alexopoulos N, Katritsis D, Raggi P. Visceral adipose tissue as a source of inflammation and promoter of atherosclerosis. Atherosclerosis. 2014;233(1):104-12.

8. Tripathy D, Mohanty P, Dhindsa S, Syed T, Ghanim H, Aljada A, et al. Elevation of free fatty acids induces inflammation and impairs vascular reactivity in healthy subjects. Diabetes. 2003;52(12):2882-7.

9. Bergman RN, Ader M. Free fatty acids and pathogenesis of type 2 diabetes mellitus. Trends Endocrinol Metab. 2000;11(9):351-6.

10. Gruzdeva O, Uchasova E, Dyleva Y, Belik E, Karetnikova V, Shilov A, et al. Multivessel coronary artery disease, free fatty acids, oxidized LDL and its antibody in myocardial infarction. Lipids Health Dis. 2014;13(1):1-9.

11. Roy VK, Kumar A, Joshi P, Arora J, Ahanger AM. Plasma free Fatty Acid concentrations as a marker for acute myocardial infarction. J Clin Diagn Res. 2013;7(11):2432-4.

12. Pilz S, Scharnagl H, Tiran B, Seelhorst $U$, Wellnitz B, Boehm BO, et al. Free fatty acids are independently associated with all-cause and cardiovascular mortality in subjects with coronary artery disease. J Clin Endocrinol Metab. 2006:91(7):2542-7.

13. Breitling LP, Rothenbacher D, Grandi NC, März W, Brenner H. Prognostic usefulness of free fatty acids in patients with stable coronary heart disease. Am J Cardiol. 2011;108(4):508-13

14. Charles MA, Fontbonne A, Thibult N, Claude JR, Warnet JM, Rosselin G, et al. High plasma nonesterified fatty acids are predictive of cancer mortality but not of coronary heart disease mortality: results from the Paris Prospective Study. Am J Epidemiol. 2001;153(3):292-8.

15. Pirro M, Mauriege P, Tchernof A, Cantin B, Dagenais GR, Després JP, et al. Plasma free fatty acid levels and the risk of ischemic heart disease in men: prospective results from the Quebec Cardiovascular Study. Atherosclerosis. 2002;160(2):377-84

16. Li S, Zhu CG, Guo YL, Xu RX, Zhang Y, Sun J, et al. The Relationship between the Plasma PCSK9 Levels and Platelet Indices in Patients with Stable Coronary Artery Disease. J Atheroscler Thromb. 2014;22(1):76-84.

17. Xu RX, Li S, Zhang Y, Li XL, Guo YL, Zhu CG, et al. Relation of plasma PCSK9 levels to lipoprotein subfractions in patients with stable coronary artery disease. Lipids Health Dis. 2014;13(1):1-9.

18. de Jongh RT, Serné EH, ljzerman RG, de Vries G, Stehouwer CD. Free fatty acid levels modulate microvascular function: relevance for obesityassociated insulin resistance, hypertension, and microangiopathy. Diabetes. 2004:53(11):2873-82.

19. Hendrickson SC, St Louis JD, Lowe JE, Abdel-aleem S. Free fatty acid metabolism during myocardial ischemia and reperfusion. Mol Cell Biochem. 1997;166(1):85-94.

20. Carlsson M, Wessman Y, Almgren P, Groop L. High levels of nonesterified fatty acids are associated with increased familial risk of cardiovascular disease. Arterioscler Thromb Vasc Biol. 2000;20(6):1588-94.

21. Westphal S, Gekeler GH, Dierkes J, Wieland H, Luley C. A free fatty acid tolerance test identifies patients with coronary artery disease among individuals with a low conventional coronary risk profile. Heart Vessel. 2002: 16(3):79-85

22. Marazzi G, Wajngarten M, Vitale C, Patrizi R, Pelliccia F, Gebara O, et al. Effect of free fatty acid inhibition on silent and symptomatic myocardial ischemia in diabetic patients with coronary artery disease. Int J Cardiol. 2007;120(1):79-84.

23. Lv ZH, Ma P, Luo W, Xiong H, Han L, Li SW, et al. Association between serum free fatty acid levels and possible related factors in patients with type 2 diabetes mellitus and acute myocardial infarction. BMC Cardiovasc Disor. 2014;14(1):159.

24. Ralston JC, Zulyniak MA, Nielsen DE, Clarke S, Badawi A, Elsohemy A, et al. Ethnic-and sex-specific associations between plasma fatty acids and markers of insulin resistance in healthy young adults. Nutr Metab. 2013;10(1):42.

25. Khawaja O, Bartz TM, Ix JH, Heckbert SR, Kizer JR, Zieman SJ, et al. Plasma free fatty acids and risk of atrial fibrillation (from the Cardiovascular Health Study). Am J Cardiol. 2012;110(2):212-6.

26. Havmoeller R, Reinier K, Teodorescu C, Ahmadi N, Kwok D, Uyevanado A, et al. Elevated plasma free fatty acids are associated with sudden death: a prospective community-based evaluation at the time of cardiac arrest. Heart Rhythm. 2014;11(4):691-6.

27. Rupp H, Zarain-Herzberg A, Maisch B. The use of partial fatty acid oxidation inhibitors for metabolic therapy of angina pectoris and heart failure. Herz. 2002:27(7):621-36.

28. Djoussé L, Benkeser D, Arnold A, Kizer JR, Zieman SJ, Lemaitre RN, et al. Plasma free fatty acids and risk of heart failure: the cardiovascular health study. Circ Heart Fail. 2013;6(5):964-9. 
29. Øie E, Ueland T, Dahl CP, Bohov P, Berge C, Yndestad A, et al. Fatty acid composition in chronic heart failure: low circulating levels of eicosatetraenoic acid and high levels of vaccenic acid are associated with disease severity and mortality. J Intern Med. 2011;270(3):263-72.

30. Pilz S, Scharnagl H, Tiran B, Wellnitz B, Seelhorst U, Boehm BO, et al.

Elevated plasma free fatty acids predict sudden cardiac death: a 6.85-year follow-up of 3315 patients after coronary angiography. Eur Heart J. 2007; 28(22):2763-9.

31. Miedema MD, Maziarz M, Biggs ML, Zieman SJ, Kizer JR, Ix JH, et al. Plasmafree fatty acids, fatty acid-binding protein 4 , and mortality in older adults (from the Cardiovascular Health Study). Am J Cardiol. 2014;114(6):843-8.

Submit your next manuscript to BioMed Central and we will help you at every step:

- We accept pre-submission inquiries

- Our selector tool helps you to find the most relevant journal

- We provide round the clock customer support

- Convenient online submission

- Thorough peer review

- Inclusion in PubMed and all major indexing services

- Maximum visibility for your research

Submit your manuscript at www.biomedcentral.com/submit
Biomed Central 